\title{
Fault Diagnosis and Reconfigurable Control of Singularly Perturbed Systems using GIMC Structure
}

\author{
Adel Tellili \\ Research unit MACS \\ ISET Djerba \\ University of Gabès, Tunisia
}

\author{
Mohamed N. Abdelkrim \\ Research unit MACS \\ Engineering school, Gabès \\ University of Gabès, Tunisia
}

\begin{abstract}
This paper proposes the extension of generalized internal model control (GIMC) based diagnosis and fault tolerant control to singularly perturbed system. This control method consists of two parts: a controller which guaranties nominal performance and works in the fault free case and a robustness controller to compensate the fault when a sensor failure is detected. This work describes the way to design such controllers for singularly perturbed systems so that the performance with the nominal controller may be guaranteed in the case of sensor failure.
\end{abstract}

\section{Keywords}

GIMC, singularly perturbed systems, coprime factorization, fault tolerant control.

\section{INTRODUCTION}

Dynamics of many physical systems contain the interaction of slow and fast phenomena. Such systems are called singularly perturbed systems. Singular perturbation problems involving several small parameters can be found in many areas like engineering and applied mathematics communities. This is due to the difficulty of these problems and in part to their wide applicability in power system dynamics, control of large scale systems, chemical reactors and similar settings. Small singular perturbation parameters typically represent unmodelled parasitic capacitances, time and inertia constants .... Controller design for such systems often suffers from high dimensionality and illconditioning, it is considerably simplified if a decomposition of fast and slow dynamics can be achieved $[1,2,3,4,5]$. Those systems, like other automated systems, are vulnerable to faults which must be detected and isolated; when a fault occurs in the system, the controller, the sensor or in the actuator, it can be amplified by the closed-loop control systems, and causes malfunction of the loop $[6,7,8,9,10]$. In order to overcome the limitation of conventional feedback, fault tolerant control systems are implemented in order to tolerate the presence of faults $[11$, 12]. There are two main classes of fault tolerant control approaches: active and passive methods. In active approaches, the principle is to use a block to detect and isolate faults in the control system. Once the diagnosis block detects and isolates a fault, the parameters and eventually the structure of the reconfigurable controller will be modified to guarantee the nominal system performance. In the passive approach, also called reliable control, a fixed controller is designed taking into account the characteristics of the fault. Usually, robust control techniques will be used without the need for system reconfiguration and fault detection [13, 14]. Several approaches have been developed to design a reliable controller, such that the resulting design guarantees closed-loop stability not only when all control components are operational, but also when faults occur $[15,16$, 17, 18, 19, 20]. Veillette (1995) employed the algebraic Riccati equation approach to develop a procedure for the design of statefeedback controllers, which could tolerate the outage within a selected subset of actuators while retaining the stability and the known quadratic performance bound. Yang et al. (2001) and Veillette et al. (1992) studied the design of controllers that could guarantee locally asymptotic stability and $\mathrm{H}_{\infty}$ performance even when some components failed. This method was extended in [21, 22] to the study of reliable control of SPS. Li et al. (2002) studied in [23] the reliable linear quadratic state-feedback control for two time scale singularly perturbed systems using slow and fast subsystems to design composite control law stabilising the fullorder closed loop system despite actuator outages. The existing approaches to the design of fault-tolerant controllers are mostly based on robust control techniques. A single controller is usually designed using robust control methods by assuming the possible failures as model uncertainties. The resulting controller is always active in the presence or absence of faults. This is the worst case design which may perform poorly compared with a non-fault tolerant control system when there are no failures [24, 25]. Zhou et al. (2001) propose new controller architecture called generalized internal model control (GIMC) and based on coprime factorization. The performance controller make it possible to maintain the system performances in the model uncertainties and external disturbances case; the robustification controller will then be active when there are model uncertainties or external disturbances. This architecture is then applied to the fault tolerant control so that the first controller is designed to satisfy the system performance and the second will be active if faults occur. Campos-Delgado (2003) et al. applied the GIMC to MIMO systems and tested this structure to a gyroscope experiment. The main goal of this work is to extend results in $[24,25]$ to the study of the fault tolerant control problem for singularly perturbed systems.

\section{MODELLING AND REDUCTION OF TWO TIME SCALES SINGULARLY PERTURBED SYSTEMS}

The singularly perturbed systems are systems presenting a strong inter-connection between their dynamics. Their models, in a continuous case, have the following form [26]:

$$
\left\{\begin{array}{c}
{\left[\begin{array}{c}
\dot{x}_{1} \\
\mu \\
\dot{x}_{2}
\end{array}\right]=A\left[\begin{array}{l}
x_{1} \\
x_{2}
\end{array}\right]+B u} \\
y=C\left[\begin{array}{l}
x_{1} \\
x_{2}
\end{array}\right]+D u
\end{array}\right.
$$

where: 
$A=\left[\begin{array}{ll}A_{11} & A_{12} \\ A_{21} & A_{22}\end{array}\right], B=\left[\begin{array}{l}B_{1} \\ B_{2}\end{array}\right]$ and $C=\left[\begin{array}{ll}C_{1} & C_{2}\end{array}\right]$

$x_{1} \in \mathfrak{R}^{n 1}, x_{2} \in \mathfrak{R}^{n 2}, u \in \mathfrak{R}^{p}, y \in \mathfrak{R}^{m}, x_{1}\left(t_{0}\right)=x_{10}$ and $x_{2}\left(t_{0}\right)=x_{20}$. $x_{1}(t)$ and $x_{2}(t)$ are respectively the slow variables and the fast variables of the system and $\mu$ is a positive scalar taking values between 0 and 1 . Two examples of physical systems being able to be modelled using the singularly perturbed systems are, on the one hand, electric motors with their loads and, on the other hand, robots with flexible articulations.

The SPS presenting two time scales can be de-coupled in two sub-models associated with the fast variables and slow variables in order to simplify the analysis and synthesis of high-ordered systems. The procedure of reduction is carried out by neglecting the fast transients against the variation time of dominant slow variables. The slow variables determine the dynamics of the system [27].

The slow subsystem (SS) is obtained by considering that the fast variables in $\mathrm{x}_{2}$ reached their established regime, which corresponds to the assumption $\mu=0$. This leads then to the following slow subsystem of dimension [26]:

$$
\left\{\begin{array}{l}
\frac{d x_{1 l}}{d t}=A_{l} x_{1 l}+B_{l} u_{l} \\
y_{l}=C_{l} x_{1 l}+D_{l} u_{l}
\end{array}\right.
$$

where:

$A_{l}=A_{11}-A_{12} A_{22}^{-1} A_{21}, B_{l}=B_{1}-A_{12} A_{22}^{-1} B_{2}$,

$C_{l}=C_{1}-C_{2} A_{22}^{-1} A_{21}$ and $D_{l}=D-C_{2} A_{22}^{-1} B_{2}$

If $A_{22}$ is non-singular, the slow model provides an approximation of the SPS behaviour. This will be valid for $t \geq t_{0}$ if the following approximation is considered $x_{1 l}\left(t_{0}\right) \approx x_{10}$.

The fast subsystem is obtained by assuming that the slow variables are constant during fast transients. This leads then to the following reduced model of dimension $n_{2}$ :

$$
\left\{\begin{array}{l}
\mu \dot{x}_{2 r}=A_{22} x_{2 r}+B_{2} u_{r} \\
y_{r}=C_{2} x_{2 r} \\
x_{2 r}\left(t_{0}\right)=x_{20}+A_{22}^{-1} A_{21} x_{1 l}\left(t_{0}\right)
\end{array}\right.
$$

where $u_{r}$ and $u_{l}$ are respectively the fast and the slow parts of the input $u$, so that : $u=u_{r}+u_{l}$.

The parameterization of the singularly perturbed system GIMC makes use of stable coprime factorization studied in [2].

\section{SPS COPRIME FACTORIZATION}

Consider the following linear time invariant SPS

$$
\left\{\begin{array}{l}
\dot{X}=A(\mu) X+B(\mu) u \\
y=C X+D u
\end{array}\right.
$$

where $\quad X=\left[\begin{array}{l}x_{1} \\ x_{2}\end{array}\right], \quad A=\left[\begin{array}{cc}A_{11} & A_{12} \\ \frac{A_{21}}{\mu} & \frac{A_{22}}{\mu}\end{array}\right], \quad B=\left[\begin{array}{c}B_{1} \\ \frac{B_{2}}{\mu}\end{array}\right]$ $C=\left[\begin{array}{ll}C_{1} & C_{2}\end{array}\right]$

The transfer function $G(s, \mu)$ of this system is:
$G(s, \mu)=C(s I-A(\mu))^{-1} B(\mu)+D$

where ' $s$ ' is Laplace variable.

$G(s, \mu)$ is two-frequency scale transfer function (TFSTF) if each term can be expanded as [2] :

$$
g(s, \mu)=\frac{n_{1}(s, \mu)+s^{k} n_{2}(\mu s, \mu)}{d_{1}(s, \mu)+s^{k} d_{2}(\mu s, \mu)}
$$

where

a- $n_{1}, n_{2}, d_{1}$ and $d_{2}$ are polynomials with coefficients analytic at $\mu=0$.

b- $\quad \operatorname{deg} d_{1}(s, \mu)=\operatorname{deg} d_{1}(s, O)=k$

c- $\operatorname{deg} d_{2}(p, \mu)=\operatorname{deg} d_{2}(p, O)$

d- $\operatorname{deg} n_{1}(s, \mu) \leq k$

e- $\operatorname{deg} n_{2}(p, \mu) \leq \operatorname{deg} d_{2}(p, \mu)$

f- the constant term of $n_{2}(p, \mu)$ and $d_{2}(p, \mu)$ are both zero.

The transfer functions of the slow and fast submodels are respectively expressed as:

$$
G_{l}(s)=C_{l}\left(s I-A_{l}\right)^{-1} B_{l}+D_{l}=G(s, 0)
$$

and

$$
G_{r}(p=\mu s)=C_{2}\left(s I-A_{22}\right)^{-1} B_{2}+D=\left.G\left(\frac{p}{\mu}, \mu\right)\right|_{\mu=0}
$$

where $p=\mu s$ is the high-frequency scale corresponding to the fast time scale $\tau=t / \mu$ in the time domain representation. The transfer functions $G_{l}(s)$ and $G_{r}(p)$ are called the low-frequency (slow) and high-frequency (fast) approximations of $G(s, \mu)$, respectively. The TFSTF $G(s, \mu)$ can be approximated by [2]:

$G(s, \mu) \approx G_{l}(s)+G_{r}(p)-G_{l}(\infty)$

where

$G_{l}(\infty)=G_{r}(0)$

Next, the coprime factorizations of the slow subsystem, fast subsystem and the full SPS will be defined.

The slow and fast subsystems have the state-space realizations $\left(A_{l}, B_{l}, C_{l}, D_{l}\right)$ and $\left(A_{22}, B_{2}, C_{2}, D\right)$, repectively. Assuming that $\left(A_{l}, B_{l}\right)$ and $\left(A_{22}, B_{2}\right)$ are stabilizable and $\left(C_{l}, A_{l}\right)$ and $\left(C_{2}, A_{22}\right)$ are detectable. There exist matrices $F_{l}, K_{l}, F_{2}$ and $K_{2}$ such that $\left(A_{l}-B_{l}\right.$ $\left.F_{l}\right),\left(A_{l}-K_{l} C_{l}\right),\left(A_{22}-B_{2} F_{2}\right)$ and $\left(A_{22}-K_{2} C_{2}\right)$ are Hurwitz matrices. For the slow subsystem, define

$$
\begin{aligned}
& M_{l}=I-F_{l}\left(s I-\left(A_{l}-B_{l} F_{l}\right)\right)^{-1} B_{l}, \\
& N_{l}=C_{l}\left(s I-\left(A_{l}-B_{l} F_{l}\right)\right)^{-1} B_{l}+D_{l} M_{l}, \\
& \tilde{M}_{l}=I-C_{l}\left(s I-\left(A_{l}-K_{l} C_{l}\right)\right)^{-1} K_{l}, \\
& \tilde{N}_{l}=C_{l}\left(s I-\left(A_{l}-K_{l} C_{l}\right)\right)^{-1} B_{l}+\tilde{M}_{l} D_{l} \\
& Y_{l}=I+F_{l}\left(s I-\left(A_{l}-K_{l} C_{l}\right)\right)^{-1} B_{l}-X_{l} D_{l}, \\
& \tilde{X}_{l}=F_{l}\left(s I-\left(A_{l}-B_{l} F_{l}\right)\right)^{-1} K_{l}, \\
& \tilde{Y}_{l}=I+C_{l}\left(s I-\left(A_{l}-B_{l} F_{l}\right)\right)^{-1} K_{l}-D_{l} \tilde{X}_{l}, \\
& X_{l}=F_{l}-\left(s I-\left(A_{l}-K_{l} C_{l}\right)\right)^{-1} K_{l},
\end{aligned}
$$

and Then the coprime factorization of the slow subsystem is:

$G_{l}(s)=N_{l} M_{l}^{-1}=\tilde{M}_{l}^{-1} \tilde{N}_{l}$

where all eight matrices are stable and satisfy the generalized Bezout identity 


$$
\left[\begin{array}{cc}
Y_{l} & X_{l} \\
-\tilde{N}_{l} & \tilde{M}_{l}
\end{array}\right]\left[\begin{array}{cc}
M_{l} & -\tilde{X}_{l} \\
N_{l} & \tilde{Y}_{l}
\end{array}\right]=\left[\begin{array}{cc}
I & 0 \\
0 & I
\end{array}\right]
$$

In the same way the coprime factorization of the fast subsystem and the full SPS are obtained as following:

$$
G_{r}(p)=N_{r} M_{r}^{-1}=\tilde{M}_{r}^{-1} \tilde{N}_{r}
$$

and

$$
G(s, \mu)=N(s, \mu) M^{-1}(s, \mu)=\tilde{M}^{-1}(s, \mu) \tilde{N}(s, \mu)
$$

The selection of matrices $\mathrm{F}$ and $\mathrm{K}$ such that $(A-B F)$ and $(A-K$ $C)$ are Hurwitz matrices is a pole placement problem which has been thoroughly investigated for singularly perturbed systems [4]. It is well known that if $F_{l}, K_{l}, F_{2}$ and $K_{2}$ such that $\left(A_{l}-B_{l} F_{l}\right),\left(A_{l}\right.$ $\left.K_{l} C_{l}\right),\left(A_{22}-B_{2} F_{2}\right)$ and $\left(A_{22}-K_{2} C_{2}\right)$ are Hurwitz matrices, then $\mathrm{F}$ and $\mathrm{K}$ are chosen as [4]:

$$
F=\left[\begin{array}{ll}
F_{1} & F_{2}
\end{array}\right] \text { and } K=\left[\begin{array}{c}
K_{1} \\
K_{2} / \mu
\end{array}\right]
$$

where

$$
F_{1}=\left(I-F_{2} A_{22}^{-1} B_{2}\right) F_{l}+F_{2} A_{22}^{-1} A_{21}
$$

and

$$
K_{1}=K_{l}\left(I-C_{2} A_{22}^{-1} K_{2}\right)+A_{12} A_{22}^{-1} K_{2}
$$

\section{RESIDUAL GENERATION}

If a plant model is available, we use the redundancy of information provided by the latter to generate signals, called residuals, carrying information on the faults. The residual should remain close to zero in fault-free case and become different from zero when a fault appears.

In presence of fault $d(t)$, the output becomes :

$$
y(s)=G(s, \mu) u(s)+G_{d}(s) d(s)
$$

Where $G_{d}(s)$ is the transfer function between the output and the fault. Using the equation (14), the relation (17) will be transformed as:

$$
y(s)-\tilde{M}^{-1}(s, \mu) \tilde{N}(s, \mu) u(s)=G_{d}(s) d(s)
$$

After multiplying the above equation with $\tilde{M}(s, \mu)$ :

$$
\tilde{M}(s, \mu) y(s)-\tilde{N}(s, \mu) u(s)=\tilde{M}(s, \mu) G_{d}(s) d(s)
$$

In the fault free case, the expression keeps a null value; in the occurrence of fault, the expression will be different from zero. Then the following residual can be defined:

$$
\begin{aligned}
r(s, \mu) & =\tilde{M}(s, \mu) y(s)-\tilde{N}(s, \mu) u(s) \\
& =\tilde{M}(s, \mu) G_{d}(s) d(s)
\end{aligned}
$$

The principle is illustrated in the following figure:

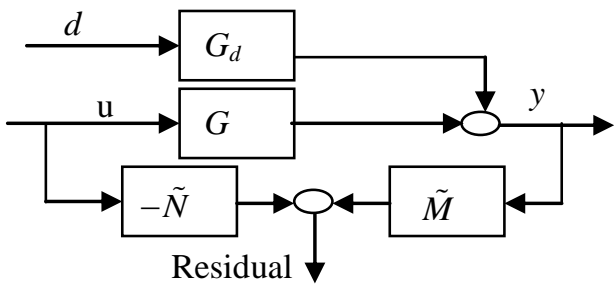

Fig 1 : Residual generation

To generate a diagnosis algorithm independent of the singularly perturbation parameter $\mu$, we will use the slow parts of the coprime factors, such that [4] :
$\left\{\begin{array}{c}\left.\tilde{M}(s, \mu)\right|_{\text {slow }}=\tilde{M}_{l}(s) \tilde{M}_{r}(0) \\ \left.\tilde{N}(s, \mu)\right|_{\text {slow }}=\tilde{N}_{l}(s) \tilde{N}_{r}(0)\end{array}\right.$

$\left.\tilde{M}(s, \mu)\right|_{\text {slow }}$ and $\left.\tilde{N}(s, \mu)\right|_{\text {slow }}$ are slow parts of $\tilde{M}(s, \mu)$ and $\tilde{N}(s, \mu)$, respectively. $\tilde{M}_{l}(s)$ and $\tilde{N}_{l}(s)$ are the slow subsystem coprime factors; $\tilde{M}_{r}(s)$ et $\tilde{N}_{r}(s)$ are the fast subsystem coprime factors. An approximation of the residual $r(s, \mu)$ is given by :

$r(s)=\tilde{M}_{l}(s) \tilde{M}_{r}(0) y(s)-\tilde{N}_{l}(s) \tilde{N}_{r}(0) u(s)$

The residual $r(s)$ is independent of the singularly perturbation parameter $\mu$, it is a good approximation of the residual $r(s, \mu)$.

\section{FTC FOR SINGULARLY PERTURBED SYSTEMS USING GIMC}

This control structure was developed by $[24,25]$ to resolve the performance and robustness problems, then it was applied to fault tolerant control. This control system consists of two parts: a nominal performance controller which works in the fault free case and a fault tolerant controller to compensate the effect of a sensor failure. The reconfiguration step is guaranteed by adding a fault tolerant loop to overcome the fault effect. The method for linear systems is presented below.

Consider the linear plant $P$ with a nominal model $P_{0}$ and $K_{0}$ the corresponding linear stabilizing controller. Define the following coprime factorizations of $K_{0}$ and $P_{0}: \quad K_{0}=\tilde{V}^{-1} \tilde{U}$ and $P_{0}=\tilde{M}^{-1} \tilde{N}$. Then all controllers $K_{0}$ that internally stabilizes $P_{0}$ can be represented as following: $K=(\tilde{V}-Q \tilde{N})^{-1}(\tilde{U}+Q \tilde{M}) \quad$ such that $\operatorname{det}(\tilde{V}(\infty)-Q(\infty) \tilde{N}(\infty)) \neq 0 \quad$ for $\quad Q \in H_{\infty} . \quad$ This new controller parameterization called GIMC is illustrated in figure 2 and proposed in $[24,25]$. The controller $Q(s)$ will be active only in the fault occurrence, i.e., when the residual signal defined in (22) is different from zero. Other ways, the system will be controlled using the performance controller $K_{0}$.

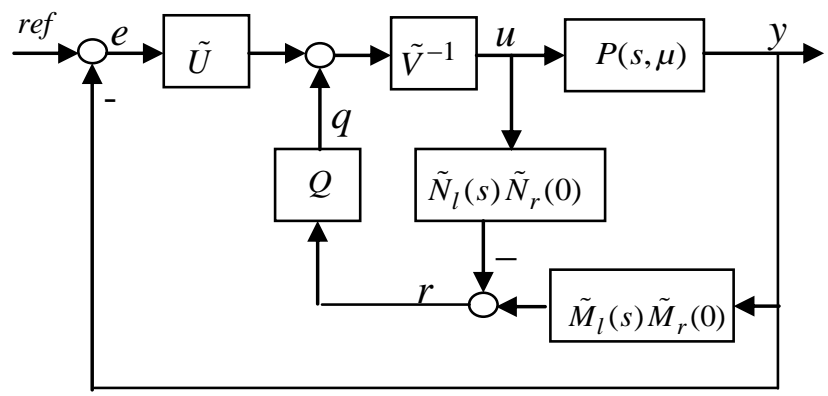

Fig 2. FTC based on GIMC structure.

The GIMC structure will be now extended to fault tolerant control of singularly perturbed systems. Consider $P(s, \mu)$ a TFSTF of the singularly perturbed system (1):

$P(s, \mu)=C(s I-A(\mu))^{-1} B(\mu)+D$

It is shown in [2] that the coprime factors of $P(s, \mu)$ are:

$P(s, \mu)=N(s, \mu) M^{-1}(s, \mu)=\tilde{M}^{-1}(s, \mu) \tilde{N}(s, \mu)$ 
and there exists $\mu^{*} \succ 0$ such that for all positive $\mu \prec \mu^{*}$, the high-frequency transfer function approximation coincides with the corresponding transfer function of the coprime factorization of the fast model (3), i.e.,

$$
\left.\tilde{N}(s, \mu)\right|_{f a s t}=\tilde{N}_{r}(p)
$$

and

$$
\left.\tilde{M}(s, \mu)\right|_{\text {fast }}=\tilde{M}_{r}(p)
$$

and Slow and fast parts of the coprime factors can be obtained using coprime factors of slow and fast subsystems, i.e.,

$\left.\tilde{M}(s, \mu)\right|_{\text {slow }}=\tilde{M}_{l}(s) \tilde{M}_{r}(0)$

and

$$
\left.\tilde{N}(s, \mu)\right|_{\text {slow }}=\tilde{N}_{l}(s) \tilde{N}_{r}(0)
$$

$\left.\tilde{M}(s, \mu)\right|_{\text {slow }}$ and $\left.\tilde{N}(s, \mu)\right|_{\text {slow }}$ are slow parts of $\tilde{M}(s, \mu)$ and $\tilde{N}(s, \mu)$, respectively. $\left.\tilde{N}(s, \mu)\right|_{\text {fast }}$ and $\left.\tilde{M}(s, \mu)\right|_{\text {fast }}$ are fast parts of $\tilde{M}(s, \mu)$ and $\tilde{N}(s, \mu)$, respectively.

An approximation $P_{0}(s)$ of the TFSTF $P(s, \mu)$ will be considered such that:

$$
\begin{aligned}
P_{0}(s) & =\left.\left.N(s, \mu)\right|_{\text {slow }} M^{-1}(s, \mu)\right|_{\text {slow }} \\
& =\left.\left.\tilde{M}^{-1}(s, \mu)\right|_{\text {slow }} \tilde{N}(s, \mu)\right|_{\text {slow }}
\end{aligned}
$$

This approximation $P_{0}(s)$ of $P(s, \mu)$ allows the calculation of the stabilising controller $K_{0}$ with the following coprime factorization:

$$
K_{0}=Y^{-1} X
$$

The set of all admissible controllers that stabilize $P(s, \mu)$ such that the closed loop transfer function is a stable TFSTF is given by [2]:

$$
\begin{aligned}
& K=(Y-Q \tilde{N})^{-1}(X+Q \tilde{M}) \\
& Q(s, \mu) \quad \text { is stable } \quad \text { TFSTF matrix } \\
& \operatorname{det}(Y(s, \mu)-Q(s, \mu) \tilde{N}(s, \mu)) \neq 0 .
\end{aligned}
$$

Thus, the fault tolerant controller based on the GIMC for singularly perturbed systems can be designed as following:

a- Design $K_{0}$ to satisfy the system performance of $P_{0}$ by assuming no faults.

b- Design $Q$ to tolerate faults.

$Q$ is active only when the residual signal is different from zero. $Q$ can be designed using passive or active fault tolerant control methods.

\section{CONCLUSION}

In this note, we studied the fault diagnosis of singularly perturbed systems using slow and fast subsystems. The so generated residual is used to build a GIMC based fault tolerant control structure to singularly perturbed systems. The controller design for performance and fault tolerance is separated. This structure operates in frequency domain; coprime factors of singularly perturbed systems were developed and approached using slow and fast subsystem coprime factors. Thus, the nominal model, used to design the performance controller, was suggested as the approximation of the full system.

\section{REFERENCES}

[1] H. K., Khalil, "Stabilization of multiparameter singularly perturbed systems", IEEE Trans. on Automatic Control, vol. AC-24, No. 5, pp. 790-791, October 1979.

[2] H. K. Khalil," Output feedback control of linear two-timescale systems", IEEE Transactions on Automatic Control, Vol. AC-32, No.9, pp. 784-792, 1987.

[3] E. H. Abed,' Decomposition and stability of multiparameter singular perturbation problems", IEEE transactions on Automatic Control, AC 31, pp. 925-934, 1986.

[4] P. Y. Kokotovic, H. K. Khalil, and J. O'Reilly, Singular perturbation methods in control: analysis and design, Academic press, Harcourt Brace Jovanovich publishers, 1986.

[5] Z. Gajic, and M. Lim. Optimal control of singularly perturbed linear systems and applications Marcel Dekker, Inc., New York, Basel, 2001.

[6] A. Tellili, M. N. Abdelkrim and M. Benrejeb. " On the fault diagnosis of uncertain singularly perturbed systems", to appear in Journal of Systems and Control Engineering.

[7] A. Tellili, M. N. Abdelkrim and M. Benrejeb. 'Sensor fault diagnosis of singularly perturbed systems applied to F-8 flight dynamic", 16th IFAC symposium on Automatic Control in Aerospace, IFAC-ACA'2004, June 14-18 2004, Saint Petersburg, Russia.

[8] H. Oloomi, M. Saif and B. Shafai, 'On the well posedness of singularly perturbed fault detection filters", in proceedings of the 2004 American Control Conference, Boston Massachusetts, June 30-july 2, 2004, pp. 5676-5677.

[9] M. Blanke, M. Staroswiecki, and N. E. Wu, "Concepts and methods in fault-tolerant control", Proceedings of American Control Conference, June 2001, Washington.

[10] M. Blanke, M. Kinneaert, J. Lunze, M. Staroswiecki, Diagnosis and fault tolerant control, Springer, 2003.

[11] H. Noura, Méthodes d'accommodation aux défauts : théorie et application. Habilitation à diriger des recherches, université Henri poincaré, Nancy 1, 26 mars 2002, France.

[12] R.J. Patton, "Fault-tolerant control systems : the 1997 situation', Safe'process, united kingdom, August 1997, pp. 1033-1055, 1997.

[13] H. Noura, D. Sauter, F. Hamelin, and D. Theilliot, 'Fault tolerant control in dynamic systems: Application to a winding machine', IEEE control Systems Magazine, Vol. 20, No. 1, pp. 33-49, 2000.

[14] J. Stoustrup, and D. Blondel, 'Fault tolerant control : A simultaneous stabilization result", IEEE transactions on automatic control, Vol. 49, No. 2, pp. 305-310, 2004

[15] F. Liao, J. L. Wang, and G. -H Yang,'Reliable robust flight tracking control : An LMI approach ", IEEE transactions on control systems technology, Vol. 10, No. 1, pp. 76-89, 2002 .

[16] R. J. Veillette, J. V. Medanić and W. R. Perkins, "' Design of reliable control systems", IEEE Transactions on Automatic Control, Vol. 37, No. 3, pp. 290-304, 1992.

[17] R. J. Veillette, 'Reliable linear-quadratic state-feedback control', Automatica, Vol. 31, pp. 137-143, 1995. 
[18] G.-H. Yang, S. -Y. Zhang, J. Lam, and J. L. Wang, "Reliable control using redundant controllers", IEEE Transactions on Automatic Control, Vol. 43, N $\mathrm{N}^{ }$. 11, pp. 1588-1593, 1998.

[19] G.-H. Yang, J. L. Wang, and Y. C. Soh, 'Reliable $\mathrm{H}_{\infty}$ controller design for linear systems", Automatica. Vol. 37, pp. 717-725, 2001

[20] Y. W. Liang, D. C. Liaw, and T. C. Lee, 'Reliable Control of Nonlinear Systems", IEEE Transactions on Automatic Control, Vol. 45, N ${ }^{\circ} .4$, pp. 706-710, April 2000.

[21] A. Tellili and M. N. Abdelkrim. " Reliable $\mathrm{H}_{\infty}$ controller design for singularly perturbed systems with sensor failure", In Proceedings of IEEE International Conference on Industrial Technology, IEEE-ICIT'04, pp. 1636-1641, december 8-10 2004, Hammamet, Tunisia.

[22] A. Tellili, M. N. Abdelkrimad and M. Benrejeb. " Reliable $\mathrm{H}_{\infty}$ control of multiple time scales singularly perturbed systems with sensor failure", to appear in International Journal of Control.
[23] Y. Li, J. L. Wang, and G. H. Yang, 'Reliable linearquadratic control for singularly perturbed systems", International Journal of Systems Science, Vol. 33, $\mathrm{N}^{\circ} 12$, pp. 949-958, 2002

[24] K. Zhou, and Z. Ren. 'A new controller architecture for high performance, robust and fault-tolerant control', IEEE Transactions on Automatic control, Vol. 46, No. 10, October 2001, pp. 1613-1618.

[25] D. Campos-Delgado and K. Zhou, 'Reconfigurable fault tolerant control using GIMC structure', IEEE Transactions on Automatic control, Vol. 48, No. 5, May 2003, pp. 832838

[26] Kokotovic, P.V. and Yakel, R.A. (1972). Singular perturbation of linear regulators: basic theorems. IEEE Trans. On automatic control, vol. AC-17, N 1 Fev. 1972, pp.29-37.

[27] Abdelkrim, M. N. and Benrejeb. M. A near robust $\mathrm{H} \infty$ control for simplified models of weakly coupled systems and singularly perturbed systems. Annales Maghrébines de l'Ingénieur, vol.15, $\quad \mathrm{N}^{\circ} 1, \quad 2003, \quad$ pp. 7-37. 